\title{
How We Treat Autoimmune Small Fiber Polyneuropathy with Immunoglobulin Therapy
}

\author{
Jill R. Schofield ${ }^{a, b} \quad$ Kamal R. Chemali ${ }^{c}$ \\ ${ }^{a}$ Center for Multisystem Disease, Denver, CO, USA; ${ }^{b}$ Department of Medicine, University of Colorado Anschutz \\ Medical Campus, Aurora, CO, USA; ' Eastern Virginia Medical School, Norfolk, VA, USA
}

\section{Keywords}

Dysautonomia $\cdot$ Autoimmune $\cdot$ Intravenous

immunoglobulin · Subcutaneous immunoglobulin .

Postural tachycardia syndrome - Small fiber neuropathy ·

autonomic neuropathy

\section{Abstract}

Intravenous immunoglobulin therapy is FDA approved for the immune-mediated peripheral nerve disorders GuillainBarre syndrome, chronic inflammatory demyelinating polyneuropathy, and multifocal motor neuropathy. Immunoglobulin therapy has been used increasingly with significant efficacy in the treatment of patients with disabling autoimmune forms of dysautonomia, which are most often small fiber (autonomic and/or sensory) polyneuropathies. It is recognized by most who treat these disorders, however, that patients with autonomic dysfunction treated with intravenous immunoglobulin therapy develop aseptic meningitis or severe lingering headache more frequently than other pa-
\end{abstract}

tient populations when this therapy is dosed in the traditional fashion. We discuss our combined 27 years of experience with the use of immunoglobulin and other immune modulatory therapy in patients with autoimmune small fiber polyneuropathy.

(c) 2019 S. Karger AG, Basel

\section{Background}

Dysautonomia is an umbrella term referring to any disorder associated with dysfunction of the autonomic nervous system. The autonomic nervous system begins in the brain, descends into the spinal cord, and then out to the small fiber autonomic nerves that innervate every organ, gland, and blood vessel in the body. There are many different mechanisms by which dysautonomia may arise, but there has been increasing evidence and awareness that it may be immune-mediated in some patients. Autoimmune autonomic dysfunction may be central, for ex-

\section{KARGER}

(c) 2019 S. Karger AG, Basel

E-Mail karger@karger.com

www.karger.com/ene
Jill R. Schofield, MD

Center for Multisystem Disease

8101 E. Lowry Blvd. Suite 250

80230 Denver, CO (USA)

E-Mail jill.schofield@ucdenver.edu 
ample, in multiple sclerosis [1], or it may be peripheral due to an immune-mediated small fiber polyneuropathy (SFPN). SFPN may manifest clinically as postural tachycardia syndrome, orthostatic intolerance, orthostatic hypotension, inappropriate sinus tachycardia, gastrointestinal dysmotility, complex regional pain syndrome, and/or neurogenic bladder [2]. In some patients, dysautonomia may occur in association with primary antineuronal autoimmunity, including antibodies to the adrenergic and muscarinic receptors, ion channels, the ganglionic acetylcholine receptor, the NMDA receptor, antibodies against the N-type or P/Q-type voltage gated calcium channels (Lambert-Eaton Syndrome), and others [3]. In other patients, it may occur in the context of systemic autoimmune disease [3, 4], and autonomic neuropathy may be the initial manifestation of Sjogren syndrome [5] or the antiphospholipid syndrome [6], which may coexist. Dysautonomia may also occur in association with most other autoimmune diseases, including some cases of Hashimoto thyroiditis [4], rheumatoid arthritis, [7] spondyloarthropathy [8], lupus [9], systemic sclerosis [10], celiac disease [11], inflammatory bowel disease [12], myasthenia gravis [13], and multiple sclerosis [1]. Indeed, it has been hypothesized that autonomic dysregulation may be involved in the etiopathogenesis of systemic autoimmunity as a result of the complex interactions between the immune system and the autonomic nervous system [14]. We recommend the use of the present protocol when administering IVIg to patients with any of these autoimmune forms of dysautonomia to reduce the likelihood of significant side effects. The only potential downside of our more gradual protocol is that the response may be delayed compared to traditional dosing, but these are chronic conditions.

Intravenous immunoglobulin (IVIg) is efficacious in the treatment of immune-mediated neuropathies, and it is FDA approved for the treatment of Guillain-Barre syndrome, chronic inflammatory demyelinating polyneuropathy, and multifocal motor neuropathy. The earliest report in the medical literature demonstrating the benefit of IVIg therapy in a patient with dysautonomia was published in the Lancet in 1996 [15]. The patient was a 23 -year-old man with "a pure dysautonomic variant" of Guillain-Barre syndrome; he was treated with IVIg with dramatic clinical improvement. Three similar reports quickly followed [16-18]. In 1999, Dupond et al. [19] reported on the dramatic efficacy of IVIg in a patient with dysautonomia and Sjogren syndrome. This was the first reported description of autonomic involvement in Sjogren syndrome. In 2013, Oaklander et al. [20] reported on

Immunoglobulin Therapy for Autoimmune SFPN the efficacy of immune modulatory therapy in children with SFPN. Ninety-eight percent had clinical evidence of dysautonomia-cardiovascular, gastrointestinal, sudomotor, and/or urological. Thirty-three percent had an underlying autoimmune disease, and $89 \%$ had 1 or more serological marker(s) suggestive of immune dysregulation. Fifteen patients were given a trial of immune modulatory therapy with IVIg and/or steroids, and 12 patients (80\%) demonstrated improvement. In 2014, Flanagan et al. [21] reported 23 patients with GI dysmotility that was presumed to be autoimmune, either because there was a comorbid autoimmune disorder, a family history of autoimmunity, or a serological abnormality suggesting autoimmunity. Nineteen of 23 patients had extraintestinal autonomic abnormalities. All patients were treated with a trial of immune modulatory therapy with 6-12 weeks of IVIg and/or methylprednisolone. Seventeen of 23 patients (74\%) improved subjectively and most also demonstrated objective improvement in autonomic testing. Most recently, Liu and Schofield separately reported 2 large retrospective case series in the use of IVIg in patients with autoimmune or "apparently autoimmune" SFPN with similar response rates of approximately $80 \%[2,22]$.

\section{Discussion}

\section{Aseptic Meningitis and Severe Prolonged Headaches}

The efficacy of IVIg therapy in a subset of patients with dysautonomia who have evidence for autonomic neuropathy by skin biopsy and/or quantitative sudomotor axon reflex testing in the context of autoantibodies that have been reported in association with autonomic dysregulation is recognized by most who treat this problem and randomized controlled studies of the use of IVIG in this population are underway [23]. It is also anecdotally recognized by most who treat these patients that this subpopulation of patients develops aseptic meningitis or severe lingering headaches significantly more often than other patient populations when IVIg is dosed in the traditional fashion, that is, $1-2 \mathrm{~g} / \mathrm{kg}$ given over $2-5$ days. Indeed, some of these patients have experienced such severe headaches or aseptic meningitis that they have refused another trial of the therapy. Giving intravenous hydration before and/or after the IVIg infusion and dividing the total monthly dose into weekly infusions, which allows for a much slower infusion rate, are usually well tolerated in these patients, and we have observed significantly fewer issues when a graded protocol is followed. A gradual uptitration of the rate with subsequent infusions 
allows the dosing frequency to be decreased eventually to monthly in the majority of patients. A suggested protocol for initiating IVIg in patients with autoimmune dysautonomia is provided in Table 1 . We estimate that at least $75 \%$ of our patients with autoimmune dysautonomias experience at least mild headache with the use of IVIg, even with the dosing modifications suggested in the present manuscript. We believe that most of these patients would experience either aseptic meningitis or severe prolonged headache with IVIg dosed in the traditional fashion.

Aseptic meningitis is an uncommon complication of IVIg given for other immune-mediated conditions. Two large retrospective studies have reported an incidence of aseptic meningitis of $0.6-1 \%$ in IVIg-treated patients [24, $25]$. Interestingly, another smaller study noted that $50 \%$ of their patients with a history of migraine developed aseptic meningitis compared to $4 \%$ of patients without a migraine history [26]. Migraine is a common comorbidity in patients with dysautonomia. The reason for the common occurrence of aseptic meningitis in patients with autonomic dysfunction and/or migraine is not known. One possible explanation is that it may occur due to IVIg-induced mast cell activation. This suspicion comes from the fact that mast cell activation syndrome is a frequent comorbidity in patients with dysautonomia, and these patients not infrequently have acute mast cell type reactions during IVIg infusions, including flushing, hives and other rashes, pruritus, and chest pain that are rapidly responsive to diphenhydramine. Furthermore, the aseptic meningitis is responsive in most patients to antihistamines, steroids, and nonsteroidal agents, each of which has mast cell inhibitory properties. Aseptic meningitis manifests clinically as severe headache, neck pain/ stiffness, nausea/vomiting, and sometimes fever. The onset is typically toward the end of the day of infusion and up to 5 days following the IVIg infusion. Cerebral spinal fluid will usually show a lymphocytic predominant pleocytosis, but a dysautonomic patient presenting with these symptoms within a few days of an IVIg infusion is much more likely to have drug-induced aseptic meningitis than infectious meningitis, and lumbar puncture and empiric antibiotics/antivirals may be avoided in most patients with this self-limited problem. Dexamethasone up to $10 \mathrm{mg}$ (or equivalent) intravenously that may be followed by a few day oral taper and/or intravenous fluids often help symptomatically when the symptoms of aseptic meningitis are severe. It should also be noted that nonsteroidal anti-inflammatory medications can rarely cause aseptic meningitis, and this is more likely in patients with autoimmune disease. This should be considered in a pa- tient receiving a stable, previously well-tolerated dosing regimen of IVIg without aseptic meningitis who later develops this complication. IVIg-induced aseptic meningitis/prolonged headache does not usually occur idiosyncratically, but rather it may occur when the infusion is given too quickly and without adequate hydration. The optimal rate of infusion may be different from patient to patient and can usually be determined by trial and error. Less severe headache and nausea (typically distinct from the patient's usual symptom complex) occur frequently with a time course similar to aseptic meningitis in dysautonomia patients treated with IVIg. Slowing the rate of infusion, decreasing the dose given with each infusion, increasing intravenous hydration, and/or the use of scheduled nonsteroidal anti-inflammatory agents, histamine 1 receptor blockers, and/or acetaminophen beginning several hours before the infusion and continuing for a few days afterwards is usually effective in this context. Premedication with steroids is also effective; however, steroids are usually used as a last resort due to their greater risk profile compared with the other options which are effective for most patients. It is also helpful for the patient to increase their oral hydration the day of and for a few days after the infusion. Triptans and dihydroergotamine may also be efficacious for these symptoms. Regarding brand of immunoglobulins, it has been our anecdotal experience that Privigen and Flebogamma are associated with more side effects in this patient population than other brands.

\section{Thrombosis}

Of note, despite all patients having autoimmune disease (a recognized thrombotic risk factor), we have not had any patient develop a thrombotic event using this specific protocol (over 100 patients total), including many patients with antiphospholipid antibodies with or without prior thrombotic events. However, all of the patients with persistent positivity for 1 or more antiphospholipid antibodies were treated with at least aspirin and some were treated with anticoagulation and/or antiplatelet therapy. One patient did develop superficial thrombophlebitis on antiplatelet therapy. This patient was switched from aspirin to apixaban and subsequent infusions were well-tolerated. We have treated patients who had a thrombotic event prior to receiving IVIg and 1 patient who had a thrombotic event using a standard IVIg protocol at another center. None of these patients was on antithrombotic therapy at the time of their clotting event and all did well with IVIg given with maintenance antithrombotic therapy. The risk of continuing 
Table 1. Sample protocol for the use of intravenous immunoglobulin in patients with autoimmune small fiber polyneuropathy

Premedications

- Methylprednisolone $60 \mathrm{mg}$ intravenously (or equivalent) before the first infusion only; occasionally, patients will need steroids with subsequent infusions due to severe mast cell activation that can be triggered by immunoglobulin therapy.

- Cetirizine $10 \mathrm{mg}$ orally before every infusion may be used up to 3 or 4 times daily for 2-3 days post-infusion for patients who develop prolonged post-infusion headaches. Other histamine-1 receptor blocking agents that are commonly used include fexofenadine, loratadine, and diphenhydramine.

- Ibuprofen (or other nonsteroidal anti-inflammatory) $400 \mathrm{mg}$ orally before infusion and 2-3 times daily for 2-3 days post-infusion for patients who develop prolonged post-infusion headaches. - Acetaminophen may be used for patients unable to take nonsteroidal anti-inflammatory agents.

Patients with documented Intravenous immunoglobulin therapy can trigger mast cell activation. This may manifest in or suspected mast cell activation syndrome any way that mast cell activation can manifest, but most commonly patients present with flushing, pruritus, hives, or other rashes. Chest pain rapidly responsive to intravenous diphenhydramine (25-50 mg), and gastrointestinal symptoms are also common. Preparation in a glass bottle as opposed to a plastic bag can reduce reactions. Some patients with severe mast cell activation syndrome take up to 8 doses of a histamine 1 receptor blocker on the day of infusion and for $2-3$ days after. Liberal dosing of histamine-2 receptor blockers, such as famotidine $20 \mathrm{mg}$ twice daily or ranitidine $150 \mathrm{mg}$ twice daily, on the day of infusion can also be helpful. Occasionally, patients will require pretreatment with steroids as above.

Product Anecdotally, Privigen and Flebogamma seem to be associated with more post-infusion side effects in this patient population. There can be variation in side effects with different products in individual patients, so if there are severe side effects, it is worth considering a trial of a different product.

Preinfusion

One liter normal saline over $1 \mathrm{~h}$, warmed to body temperature for patients with severe autonomic dysregulation and/or mast cell activation syndrome as temperature change is often a trigger for mast cell activation. If needed, a second liter can be given at the end of the IVIg infusion for patients with prolonged headaches despite the above treatments, those with a tendency toward thrombotic events or those who experience chest pain due to blood hyperviscosity produced by IVIg. Note that intravenous fluids should not run concomitantly with the IVIg due to the risk of precipitation of the antibody molecules.

Dose The starting dose should be $1 \mathrm{~g} / \mathrm{kg}$ every 28 days. The monthly dose should not change, only the infusion schedule.

$0.25 \mathrm{~g} / \mathrm{kg}$ weekly $\times 4$ infusions within 28 days then

$0.33 \mathrm{~g} / \mathrm{kg}$ approximately every 10 days $\times 3$ infusions within 28 days then

$0.50 \mathrm{~g} / \mathrm{kg}$ approximately every 14 days $\times 2$ infusions within 28 days then

$0.75 \mathrm{~g} / \mathrm{kg}$ approximately every 21 days $\times 1-2$ infusions then

$1.0 \mathrm{~g} / \mathrm{kg}$ approximately every 28 days thereafter.

Patients may advance to the next dosing schedule after 2 infusions if there are not severe post-infusion symptoms. Patients with significant post-infusion symptoms may not tolerate advancing to the next dosing interval. A continuous rate is used for the whole infusion. For the first infusion, the rate of infusion should be $3 \mathrm{~g}$ per h or the slowest possible rate that will allow completion of the infusion within the infusion staff hours. With subsequent infusions, if the patient tolerated the first infusion without severe headaches or other issues, the rate may be increased by $1 \mathrm{~g}$ per $\mathrm{h}$ per infusion. For example, for the 2 nd infusion, use a rate of $4 \mathrm{~g}$ per h. For subsequent infusions, continue to increase the continuous rate as tolerated by $1 \mathrm{~g}$ per h per infusion. If the patient develops post-infusion headache or other symptoms, initiate a histamine 1 receptor blocker 2-4 times daily and a nonsteroidal anti-inflammatory agent on a scheduled basis starting the morning of the infusion and continuing for 2-3 days post infusion. If the patient has significant side effects despite scheduled antihistamine and nonsteroidal therapy, then add $1 \mathrm{~L}$ of normal saline over $1 \mathrm{~h}$ at the end of the immunoglobulin infusion. If the patient still develops significant symptoms despite the above measures, then return to the prior infusion rate and dosing schedule and consider switching to subcutaneous immunoglobulin. 
IVIg with the addition of antithrombotic therapy in these patients must be weighed against the risk of not treating their disabling illness.

\section{IVIg Dosing, Response Rate, and Duration}

of Therapy

As noted in Table 1, our starting dose of IVIg in patients with autoimmune autonomic neuropathy is $1 \mathrm{~g} / \mathrm{kg} /$ monthly (administered in weekly divided doses). If there is not progressive improvement to a goal of at least $80 \%$ of pre-illness functional level by approximately 6-9 months of therapy, then a trial of increasing the dose incrementally up to a maximum of $2 \mathrm{~g} / \mathrm{kg}$ monthly to determine if there is an incremental benefit is worthwhile as some patients do better with a higher dose. Most of our patients respond optimally to a dose of $1-1.5 \mathrm{~g} / \mathrm{kg} / \mathrm{month}$ ly (average approximately $1.3 \mathrm{~g} / \mathrm{kg} /$ monthly). Using this protocol, patients may begin to see clinical improvement after an average of 6 weeks of therapy (range 2-12 weeks) [2]. The improvement is usually gradual. For example, the patient may notice that their "bad days" are less bad and/or their "good days" are more good. They may then note that they have fewer bad days and more good days and that in general they are able to tolerate more intensive endeavors than they were before. Improvement often continues very slowly over 6-12 months and may continue for up to 2 years. This may be due to the expected slow regeneration of the small fiber neurons. If there is no improvement after 4-6 months of continuous therapy, the treatment should be stopped.

While patients with an acute infection-induced immune-mediated autonomic neuropathy may be effectively treated with 1-2 courses of IVIg [27], patients with a more gradual and progressive disease onset in association with systemic autoimmunity almost always require a longer duration of therapy that may be indefinite. This is analogous to many other systemic autoimmune diseases, such as rheumatoid arthritis, autoimmune hepatitis, and multiple sclerosis, which often require lifelong therapy. While this has not yet been studied in autoimmune autonomic neuropathy, all but one of our patients who has experienced an interruption of therapy (for insurance or other reasons) experienced a return to their prior baseline or in some cases worse than their prior baseline (in patients who were treated earlier in their course). For all of these patients, however, the duration of treatment was less than 1 year. Dr. Liu, however, has reported 16\% of their patients were eventually able to stop IVIg [22]. Dependence on IVIg can be assessed by gradually reducing the dosing interval, for example, from every 4 weeks to every 5 weeks to every 6 weeks and so on. If there is clinical worsening, the plan should be to resume the therapy at the previously effective dose for at least another year before considering another trial of reducing the dosing interval. Approximately $50 \%$ of patients with chronic inflammatory demyelinating polyneuropathy are eventually able to stop IVIg therapy, but some patients require several years of therapy.

Our experience with the use of IVIg in patients with autoimmune autonomic neuropathy matches that which has been reported by Oaklander and Flanagan with a response rate of approximately $75-80 \%$. Many patients improve by $80-90 \%$ of their pre-illness level of functioning. It is very important for patients with orthostatic intolerance to optimize and maintain lifestyle measures, including regular strengthening and aerobic exercise, optimal salt and fluid intake, compression stockings, and elevation of the head of the bed. Some responsive patients are able to eventually decrease the doses or even discontinue their dysautonomia-specific therapies, for example, fludrocortisone or beta blockers, following a successful response to immune modulatory therapy. Others do better if they maintain some or all of their autonomic medications.

\section{Subcutaneous Immunoglobulin Therapy}

For patients with significant symptoms from IVIg despite the above measures or for patients with venous access issues, transition to subcutaneous immunoglobulin (SCIg) therapy should be considered, as it is better tolerated by many patients. SCIg therapy has been used successfully for many years in patients with immune deficiency syndromes, and increasing data have shown that it may also be effective for autoimmune disease [28]. The administration of the higher doses required to treat autoimmune disease is technically more difficult, however, requiring more subcutaneous infusion sites and more frequent infusions than is required for patients with humoral immunodeficiency syndromes.

\section{Other Immune Modulatory Agents}

Importantly, failure to respond to IVIg does not preclude a response to other immune-modulatory therapies. Alternative therapies for patients with autoimmune autonomic neuropathy include pulse high-dose steroids or chronic steroid therapy with a prolonged taper, rituximab, therapeutic plasma exchange, and oral immune modulatory agents (e.g., mycophenolic acid or azathioprine), and some patients require combination therapy. As with IVIg, therapeutic plasma exchange 
used in this context must also be given on a chronic basis, with the exchange interval determined by the duration of response to the initial 5 treatments. For example, if there is improvement for 14 days, then a dosing interval of therapeutic plasma exchange of every 7-10 days may be considered. Usually other immune modulatory agents, for example, rituximab, are then started with the goal of eventually terminating the therapeutic plasma exchange, which is not an attractive long-term option, recognizing that the optimal time to response to rituximab and other therapies in immune-mediated neurological disorders may require 1 year or even longer of therapy. For nonresponsive patients, it is also important to consider whether there may be other important comorbidities that may be seen in conjunction with autoimmune dysautonomia that are not being addressed, including diabetes mellitus or prediabetes, vitamin $\mathrm{D}, \mathrm{B} 1$, or B12 deficiency or vitamin B6 toxicity or deficiency, the presence of an M-protein, underlying mast cell activation syndrome, chronic infection, median arcuate ligament syndrome, spinal fluid leak, Chiari I malformation and/or cranio-cervical instability, and mitochondrial cytopathies.

\section{Insurance Coverage of Immunoglobulin Therapy for Autoimmune SFPN}

Since autoimmune SFPN is not currently an FDA-approved indication for IVIg, insurance approval usually requires a prior authorization. We have found it helpful to write a detailed letter summarizing the rationale and a summary of peer-reviewed articles available to date demonstrating efficacy. Oftentimes, a peer-to-peer discussion with an insurance company physician is also required. Comparing autoimmune SFPN to the other peripheral immune-mediated neuropathies that are FDA-approved indications for IVIg has been an effective strategy. We have also found it useful to explain that the ability to diagnose SFPN has lagged behind the ability to diagnose the large fiber neuropathies, which has led to fewer completed studies to date. We have also found it helpful to note that there are randomized controlled trials underway investigating the efficacy of IVIg in patients with immunemediated SFPN, to explain that patients with autoimmune dysautonomia represent only a subset of all patients with dysautonomia and to request IVIg as a 4-6 month trial to be continued only if there is significant symptomatic improvement.

\section{Conclusions}

Intravenous immunoglobulin therapy has emerged as an effective therapy in patients with autoimmune forms of dysautonomia, but when dosed in the traditional fashion, it is not as well tolerated in this patient population as it is in most other patients. Modifying the infusion protocol, however, allows most patients to tolerate this therapy with only mild to moderate side effects. SCIg therapy is an excellent alternative option for patients who have significant side effects from IVIg despite dosing modifications or for patients with venous access issues. Other treatments that are worth considering include plasma exchange, rituximab, steroids, and other oral immune modulatory agents.

\section{Disclosure Statement}

There was no sponsorship or financial support provided for this work and neither author has any conflicts of interest to disclose.

\section{References}

1 Kanjwal K, Karabin B, Kanjwal Y, Grubb BP. Autonomic dysfunction presenting as postural orthostatic tachycardia syndrome in patients with multiple sclerosis. Int J Med Sci. 2010 Mar;7:62-7.

2 Schofield JR, Chemali KR. The efficacy and safety of intravenous immunoglobulin in severe autoimmune dysautonomias: a retrospective analysis of 38 patients. Am J Ther. $2018 ; 14$.

3 Vernino S, Stiles LE. Autoimmunity in postural orthostatic tachycardia syndrome: current understanding. Auton Neurosci. 2018 Dec;215:78-82.
4 Blitshteyn S. Autoimmune markers and autoimmune disorders in patients with postural tachycardia syndrome (POTS). Lupus. 2015 Nov;24(13):1364-9.

5 Barendregt PJ, Markusse HM, Man In 't Veld AJ. Primary Sjögren's syndrome presenting as autonomic neuropathy. Case report. Neth J Med. 1998 Nov;53(5):196-200.

6 Schofield JR. Autoimmune autonomic neuropathy-in its many guises-as the initial manifestation of the antiphospholipid syndrome. Immunol Res. 2017 Apr;65(2):532-542.

7 Adlan AM, Lip GY, Paton JF, Kitas GD, Fisher JP. Autonomic function and rheumatoid arthritis: a systematic review. Semin Arthritis Rheum. 2014 Dec;44(3):283-304.

8 Borman P, Gokoglu F, Kocaoglu S, Yorgancioglu ZR. The autonomic dysfunction in patients with ankylosing spondylitis: a clinical and electrophysiological study. Clin Rheumatol. 2008 Oct;27(10): 1267-73.

9 Bortoluzzi A, Silvagni E, Furini F, Piga M, Govoni M. Peripheral nervous system involvement in systemic lupus erythematosus: a review of the evidence. Clin Exp Rheumatol. 2019 Jan-Feb;37(1):146155
Immunoglobulin Therapy for Autoimmune SFPN 
10 Adler BL, Russell JW, Hummers LK, McMahan ZH. Symptoms of Autonomic Dysfunction in Systemic Sclerosis Assessed by the COMPASS-31 Questionnaire. J Rheumatol. 2018 Aug;45(8):1145-52.

11 Gibbons CH, Freeman R. Autonomic neuropathy and coeliac disease. J Neurol Neurosurg Psychiatry. 2005 Apr;76(4):579-81.

12 Gondim FA, Oliveira GR, Teles BC, Souza $\mathrm{MH}$, Braga LL, Messias EL. A case-control study of the prevalence of neurological diseases in inflammatory bowel disease (IBD). Arq Neuropsiquiatr. 2015 Feb;73(2):11924.

13 Benjamin RN, Aaron S, Sivadasan A, Devasahayam S, Sebastin A, Alexander M. The Spectrum of Autonomic Dysfunction in Myasthenic Crisis. Ann Indian Acad Neurol. 2018 Jan-Mar;21(1):42-8.

14 Koopman FA, Tang MW, Vermeij J, de Hair MJ, Choi IY, Vervoordeldonk MJ, et al. Autonomic dysfunction precedes development of rheumatoid arthritis: a prospective cohort study. EBioMedicine. 2016 Apr;6:231-7.

15 Heafield MT, Gammage MD, Nightingale S, Williams AC. Idiopathic dysautonomia treated with intravenous gammaglobulin. Lancet. 1996 Jan;347(8993):28-9.

16 Mericle RA, Triggs WJ. Treatment of acute pandysautonomia with intravenous immu- noglobulin. J Neurol Neurosurg Psychiatry. 1997 May;62(5):529-31.

17 Smit AA, Vermeulen M, Koelman JH, Wieling $\mathrm{W}$. Unusual recovery from acute panautonomic neuropathy after immunoglobulin therapy. Mayo Clin Proc. 1997 Apr;72(4):333-5.

18 Venkataraman S, Alexander M, Gnanamuthu C. Postinfectious pandysautonomia with complete recovery after intravenous immunoglobulin therapy. Neurology. 1998 Dec; 51(6):1764-5.

19 Dupond JL, Gil H, de Wazieres B. Five-year efficacy of intravenous gammaglobulin to treat dysautonomia in Sjögren's syndrome. Am J Med. 1999 Jan;106(1):125.

20 Oaklander AL, Klein MM. Evidence of smallfiber polyneuropathy in unexplained, juvenile-onset, widespread pain syndromes. Pediatrics. 2013 Apr;131(4):e1091-100.

21 Flanagan EP, Saito YA, Lennon VA, McKeon A, Fealey RD, Szarka LA, et al. Immunotherapy trial as diagnostic test in evaluating patients with presumed autoimmune gastrointestinal dysmotility. Neurogastroenterol Motil. 2014 Sep;26(9):1285-97.

22 Liu X, Treister R, Lang M, Oaklander AL. IVIg for apparently autoimmune small-fiber polyneuropathy: first analysis of efficacy and safety. Ther Adv Neurol Disorder. 2018 Jan; 11:17562856177444484.
23 de Greef BT, Geerts M, Hoeijmakers JG, Faber CG, Merkies IS. Intravenous immunoglobulin therapy for small fiber neuropathy: study protocol for a randomized controlled trial. Trials. 2016 Jul;17(1):330.

24 Bharath V, Eckert K, Kang M, Chin-Yee IH, Hsia CC. Incidence and natural history of intravenous immunoglobulin-induced aseptic meningitis: a retrospective review at a single tertiary care center. Transfusion. 2015 Nov; 55(11):2597-605.

25 Kemmotsu Y, Nakayama T, Matsuura H, Saji T. Clinical characteristics of aseptic meningitis induced by intravenous immunoglobulin in patients with Kawasaki disease. Pediatr Rheumatol Online J. 2011 Sep;9(1):28.

26 Sekul EA, Cupler EJ, Dalakas MC. Aseptic meningitis associated with high-dose intravenous immunoglobulin therapy: frequency and risk factors. Ann Intern Med. 1994 Aug; 121(4):259-62.

27 Goodman BP. Immunoresponsive postinfectious autonomic neuropathy. Am J Ther. 2014 Jul-Aug;21(4):e120-3.

28 Sala TP, Crave JC, Duracinsky M, Lepira Bompeka F, Tadmouri A, Chassany O, et al. Efficacy and patient satisfaction in the use of subcutaneous immunoglobulin immunotherapy for the treatment of auto-immune neuromuscular diseases. Autoimmun Rev.2018Sep;17(9):873-81. 